\title{
What makes an effective engineering diagram? A comparative study of novices and experts
}

\section{Dr. Alisha A Waller, Georgia Institute of Technology}

Dr. Alisha A. Waller has been active within ASEE since 1991, serving the ERM Division in multiple capacities. She has won the Helen Plants Award three times and was awarded the Distinguished Service award in 1998. Her research interests include diversity, reflective learning, and multiple representations. Her teaching interests include optimization, probability, and statistics. She is currently affiliated with Biomedical Engineering Department at Georgia Tech.

Prof. Joseph M LeDoux, Georgia Institute of Technology

Dr. Wendy C Newstetter, Georgia Institute of Technology

Dr Wendy C. Newstetter is the Director of Educational Research and Innovation in the College of Engineering at the Georgia Institute of Technology. 


\title{
What makes an effective engineering diagram? A comparative study of novices and experts
}

\begin{abstract}
Engineers have a predictable way of working. A text $(\mathrm{T})$ or verbal description of a problem is translated into a diagram (D) which bootstraps the creation of a symbolic/mathematical (S) model for further analysis. Learning to solve problems in this particular way is a major goal for engineering education. The research presented in this paper focuses specifically on the text to diagram translation and the particularized representations utilized within a course on conservation principles. Previous research on student-generated diagrams revealed that, at the beginning of the course, students are not able to construct useful diagrams that follow the conservation laws. This result led to the general question of whether students can recognize useful, correct diagrams; more specifically: 1) Given a set of diagrams, are students able to distinguish between effective and ineffective diagrams? and 2) How do students' judgments compare with those of an expert? To answer these questions, we conducted an exploratory study of students' ranking of a set of diagrams for a complex, multi-unit problem. We compared them to those of an expert using Spearman's Rank Correlation Coefficient in a cluster analysis. These findings are complimented by results from the post-exercise class discussion regarding criteria for a good diagram and have important implications for teaching and for future research.
\end{abstract}

\section{Introduction}

Engineers have a particular, predictable way of working. Problems first encountered through text or verbal description are translated into a diagram which abstracts the salient features, highlights underlying system structure, instantiates laws and principles, and organizes the complexity of the problem. The diagram becomes a "tool for thinking", a bootstrapping mechanism which serves as the basis for creating a symbolic/mathematical model for further analysis. Learning to create and successfully employ the many engineering-community-sanctioned diagrams (e.g. free body diagrams, multi-unit flow diagrams, circuit diagrams) is a major goal for engineering students and educators alike.

The research presented in this paper is part of an extensive study on the text to diagram to symbol translations and representations utilized within a course on conservation principles. Previous research on student-generated diagrams revealed that, at the beginning of the course, students are not able to construct useful diagrams that follow the conservation laws. This result led to the general question of whether students can recognize useful, correct diagrams, which is a cognitively easier task than generating them. We operationalized this question more specifically as 1) Given a set of diagrams, are students able to distinguish between effective and ineffective diagrams? and 2) How do students' judgments compare with those of an expert?

For diagnostic purposes at the start of the term, students were given a textual description of a multi-unit process and a set of seven different diagrams. They were asked to rank order these diagrams with respect to their usefulness in further analysis of the system. This task assessed whether the students could recognize good qualities for a diagram to have. In this paper, we 
analyze the agreement between an expert and the students on the diagram rankings using Spearman's Rank Correlation Coefficient. In addition, we discuss the task in context, the ensuing class discussion regarding criteria for a good diagram, and the implications of our findings for teaching and for future research.

\section{Challenges associated with problem framing and problem solving with principles of conservation}

In Chemical Engineering and some Biomedical Engineering curricula, a course focused on principles of conservation of mass and energy is a first introduction to thinking and problem solving like an engineer. In this course students first encounter the engineering problem solving method, which depends on generating a diagram representing units in a complex process as a first step towards a numerical solution ${ }^{1}$. In generating this diagram, students need to first extract the salient elements of the process from a word problem and transform those elements into a community-sanctioned, multi-unit process diagram with appropriate notations and available data. The physical flow of materials through the units is represented by "streams" which consist of one or more "species" of matter, which can be challenging for students to identify. An essential unifying principle of this course is balance within units and across systems, a principle that students need to understand and consistently apply in solving such problems. This might seem straightforward, nevertheless, in our recent survey of students' success and failure in mass/energy balance courses in a number of institutions, we found that a significant percentage of students earned a D, F, or withdrew from this introductory course on conservation principles. Table 1 illustrates the difficulties that engineering students have with entry-level engineering courses at Georgia Tech.

\begin{tabular}{|l|c|c|c|c|}
\hline Engineering Domain & $\begin{array}{c}\text { Percent } \\
\text { D's }\end{array}$ & $\begin{array}{c}\text { Percent } \\
\text { F's }\end{array}$ & $\begin{array}{c}\text { Percent } \\
\text { W's }\end{array}$ & $\begin{array}{c}\text { Percent D's, F's, } \\
\text { and W's }\end{array}$ \\
\hline Civil / Environmental & 4 & 1 & 8 & 13 \\
\hline Chemical & 8 & 6 & 7 & 21 \\
\hline $\begin{array}{l}\text { Mechanical / Aerospace / } \\
\text { Materials }\end{array}$ & 8 & 4 & 7 & 19 \\
\hline Computer / Electrical & 5 & 3 & 8 & 16 \\
\hline Industrial & 4 & 3 & 8 & 15 \\
\hline
\end{tabular}

Table 1: Student grades in entry-level engineering courses at Georgia Tech

Table 2 focuses on entry-level conservation courses in Chemical Engineering and Biomedical Engineering at several peer institutions. The percentage of students who earn a D or an F or who withdraw from the course justifies further research on these entry-level courses. 


\begin{tabular}{|l|l|c|c|c|c|}
\hline School & Department & $\begin{array}{c}\text { Students / } \\
\text { course }\end{array}$ & $\begin{array}{c}\text { Percent } \\
\text { D's or F's }\end{array}$ & $\begin{array}{c}\text { Percent } \\
\text { W's }\end{array}$ & $\begin{array}{c}\text { Percent D's, F's, } \\
\text { and W's }\end{array}$ \\
\hline UC Berkeley & Chem Engr & 130 & 5 & 23 & 28 \\
\hline $\begin{array}{l}\text { U. of } \\
\text { Minnesota }\end{array}$ & Chem Engr & 220 & 14 & 10 & 24 \\
\hline $\begin{array}{l}\text { U. of } \\
\text { Wisconsin }\end{array}$ & Chem Engr & 95 & 12 & 1 & 13 \\
\hline Georgia Tech & $\begin{array}{l}\text { Biomed } \\
\text { Engr }\end{array}$ & 70 & 16 & 12 & 28 \\
\hline
\end{tabular}

Table 2: Student grades in selected entry-level engineering courses nationwide

With funding from the National Science Foundation, we have been investigating the transformation from a textual account of a problem to a diagrammatic representation within an expert/novice framework. Our goal is to better understand how experts generate and use diagrams informed by principles of conservation in comparison to novices new to this reasoning and problem solving strategy. In using this expert/novice framework, we subscribe to the implied hypothesis that there may be a developmental dimension to learning to solve problems in this community-sanctioned way, which has significant implications for teaching interventions. In particular, we recognize that becoming an "expert" often takes decades and hundreds of experiences. Although colleges can not reasonably expect students to move from "novice" as a first-year student to "expert" by graduation, we aim to help them make significant progress in this trajectory.

Across all engineering disciplines, faculty encounter student resistance to generating and using diagrams in problem solving. It is not uncommon to require the diagrams for full credit when grading in order to motivate students to construct them. Unfortunately, many students see diagrams as merely fulfilling a component of the assignment rather than as an essential cognitive tool. Furthermore, when students do generate diagrams, they are often ineffective and are not actually used by the students to scaffold the mathematical analysis. We define an "effective diagram" as one that is an effective cognitive tool for generating correct mathematical models of the problem, as well as being parsimonious and computationally efficient. Parsimony is reflected in using the minimally appropriate number of units, streams, and species. Computationally efficient refers to the appropriate placement of information and a consistent and meaningful notation.

Here we report on an exploratory study of novice (student) and expert (faculty) understandings of effective diagrams. The novice-expert research approach for exploring conceptual understanding and learning has been useful in illuminating important developmental issues in both physics and engineering education ${ }^{2-4}$. Observing the students' difficulties in generating diagrams led to the more general question of whether students can recognize and distinguish between effective and ineffective diagrams. From a cognitive perspective, it is much easier to recognize and distinguish than to generate, which is why we chose this study design strategy. 
The research questions we explored in this study were:

- Given a set of diagrams, are students able to distinguish between effective and ineffective diagrams?

- How do students' judgments compare with those of an expert?

\section{Methods}

We designed a ranking exercise which was administered to a class section of 44 students. The "Wash and Walk" problem was a multi-unit industrial process for washing clothes. The complete problem is given in Appendix I. Seven diagrams of a subsystem of the problem were developed which met the following five criteria in different degrees. First, the diagram must correctly represent the underlying structure of the system. Second, the diagram must obey the conservation principles. For example, in a non-reacting system, if water enters a unit, it must also leave the unit. We refer to this level of conservation as "species balance" which is a precursor to mass balance. Third, the diagram must be computationally efficient. Fourth, the notational system used to reference elements in the diagram should be consistent and meaningful, e.g. $\mathrm{W}^{\mathrm{k}}$ represents the mass of water in stream k. Finally, the diagram should not contain distractors which are not relevant to the problem scope and definition, for example, the paddle in a mixer should not be drawn.

We applied these five criteria to a subsystem of the Wash and Walk problem. The system structure of this problem is two units connected by five streams in the following way:

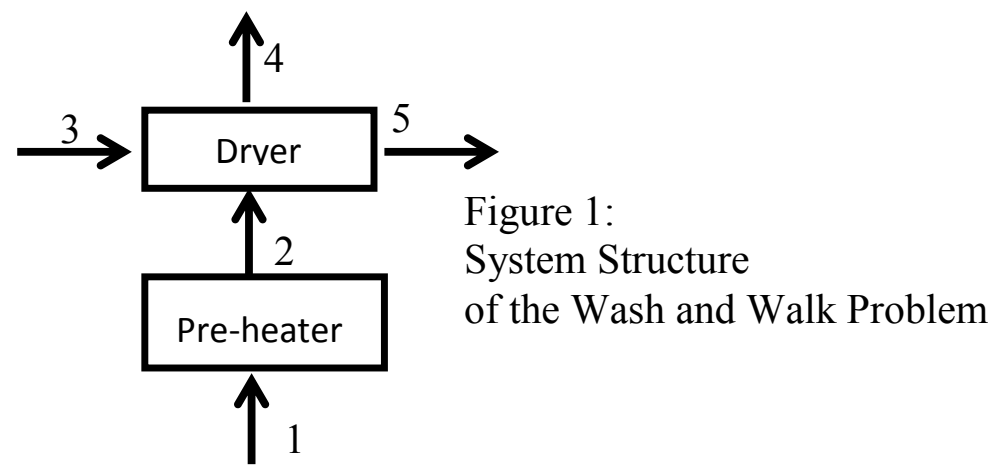

Second, species balance requires that the streams contain the following species: 1) air, water; 2) air, water; 3) water, clothes; 4) air, water; and 5) clothes. The third criterion is to be computationally efficient. In this problem, there are 10 data points given, but two express the same information ( $1 \mathrm{~atm}$ and $14.7 \mathrm{psi})$; therefore, there are 9 distinct pieces of data. Fourth, the notational system of the diagram should provide a consistent way to reference each stream in the diagram, leading to a consistent way to reference each species in each stream. Finally, the diagram should be free of distractions, e.g. the control buttons on the dryer should not be drawn because their location is irrelevant to solving the mass and energy balance problems (however, their location would not be irrelevant in solving other problems, such as safety and userfriendliness). Table 3 below summarizes the quality of the seven diagrams with respect to these criteria. 


\begin{tabular}{|l|c|c|c|c|c|c|c|}
\hline Diagram & A & B & C & D & E & F & G \\
\hline 1. Correct system structure & $\mathrm{N}$ & $\mathrm{N}$ & $\mathrm{Y}$ & $\mathrm{N}$ & $\mathrm{Y}$ & $\mathrm{N}$ & $\mathrm{Y}$ \\
\hline 2a. Species balance & $\mathrm{N}$ & $\mathrm{Y}$ & $\mathrm{Y}$ & $\mathrm{N}$ & $\mathrm{Y}$ & $\mathrm{Y}$ & $\mathrm{Y}$ \\
\hline 2b. Correct species & $\mathrm{N}$ & $\mathrm{N}$ & $\mathrm{Y}$ & $\mathrm{N}$ & $\mathrm{N}$ & $\mathrm{N}$ & $\mathrm{N}$ \\
\hline 3a. Number of data missing & 3 & 5 & 4 & 4 & 2 & 2 & 1 \\
\hline 3b. Located efficiently & $\mathrm{Y}$ & $\mathrm{Y}$ & $\mathrm{Y}$ & $\mathrm{Y}$ & $\mathrm{Y}$ & $\mathrm{Y}$ & $\mathrm{N}$ \\
\hline 4. Streams labeled & $\mathrm{N}$ & $\mathrm{Y}$ & $\mathrm{Y}$ & $\mathrm{Y}$ & $\mathrm{N}$ & $\mathrm{N}$ & $\mathrm{N}$ \\
\hline 5. Free of distractions & $\mathrm{N}$ & $\mathrm{Y}$ & $\mathrm{Y}$ & $\mathrm{N}$ & $\mathrm{Y}$ & $\mathrm{N}$ & $\mathrm{Y}$ \\
\hline
\end{tabular}

Table 3: Evaluation of Diagrams

( $\mathrm{Y}=$ meets criterion; $\mathrm{N}=$ does not meet criterion)

Combining this explicit, criteria-based analysis with tacit, experiential knowledge, the expert ranked the diagrams from best to worst as C, G, E, F, B, A, and D. The full problem description is in Appendix I and the diagrams are in Appendix II.

\section{Data Collection}

On the first day of class, the data collection proceeded as follows:

1. Students read a description of a complex, multi-unit, industrial clothes washing process and generated a visual representation (diagram, schematic, etc) of the process that would help to quantitatively evaluate its design.

2. Students compared and contrasted their representation with a partner, discussing why and how they made their representation decisions.

3. Student pairs then ranked the given seven diagrams from best to worst as to their usefulness in doing a quantitative evaluation.

4. The class engaged in a guided discussion about the diagrams, the qualities of a community-sanctioned diagram, and the diagram as a cognitive tool for thinking. In addition to collecting the student pairs' rankings, we audiotaped the entire two-hour class period and recorded our observations during the class.

\section{Data Analysis and Interpretation}

In our data analysis, we start with the quantitative data, comparing the rankings. Then we move to the qualitative data of the diagrams themselves, classroom observations, and the audio recording of the class period to see if we may uncover some explanations for the quantitative results.

\section{Initial Analysis}

The initial analysis is to examine the range of rankings for each diagram by the students. Table 4 below illustrates three interesting results. First, the first row shows that every diagram was ranked either first or second by at least one student pair, indicating a wide ranging evaluation of what constitutes an effective diagram. Second, every diagram except E was ranked worst by at least one student pair, as seen on the second row. Third, Diagram E is the only one ranked from medium quality to highest quality by all student pairs. 


\begin{tabular}{|c|c|c|c|c|c|c|c|}
\hline \multicolumn{8}{|c|}{ Diagram } \\
\hline & $\mathbf{A}$ & B & C & D & $\mathbf{E}$ & $\mathbf{F}$ & $\mathbf{G}$ \\
\hline $\begin{array}{l}\text { Highest } \\
\text { Rank by } \\
\text { Students }\end{array}$ & 2 & 2 & 1 & 2 & 1 & 1 & 1 \\
\hline $\begin{array}{l}\text { Lowest } \\
\text { Rank by } \\
\text { Students }\end{array}$ & 7 & 7 & 7 & 7 & 4 & 7 & 7 \\
\hline
\end{tabular}

Table 4: Highest and Lowest Rankings by Diagram

\section{Comparing novice rankings to the expert ranking}

The goal of the second analysis is to determine to what degree the students (novices) and the professor (expert) agree in their rankings of the diagrams. Since we are comparing the ordered rankings of seven diagrams, we chose to use Spearman's Rank Correlation Coefficient, which measures the strength of association between two ranked variables, as our statistic ${ }^{5}$. Because our sample size is relatively small $(n=7)$, we computed the exact $p$-values for each potential value of the statistic instead of using the t-distribution as an approximation. We first summarized the class rankings by averaging the rank for each diagram across the 22 student pairs. Using these averages, we created the class composite ranking. These data, the expert ranking, and highest/lowest rankings are shown in Table 5 below.

\begin{tabular}{|l|c|c|c|c|c|c|c|}
\hline \multicolumn{7}{|c|}{ Diagram } & D \\
\hline $\begin{array}{l}\text { Expert } \\
\text { Ranking }\end{array}$ & 6 & 5 & 1 & 7 & 3 & 4 & 2 \\
\hline $\begin{array}{l}\text { Average } \\
\text { Student } \\
\text { Ranking }\end{array}$ & 4.68 & 5.18 & 4.05 & 5.05 & 2.00 & 3.23 & 3.82 \\
\hline $\begin{array}{l}\text { Class } \\
\text { Composite } \\
\text { Ranking }\end{array}$ & 5 & 7 & 4 & 6 & 1 & 2 & 3 \\
\hline
\end{tabular}

Table 5: Expert and Class Composite Diagram Rankings

Comparing the expert ranking with the combined class ranking yields a Spearman's Rank Statistic of 0.571 , with a p-value of 0.083 , which is not statistically significant. Therefore, we fail to reject the null hypothesis that the two rankings have no association. In other words, we fail to find evidence that the combined class ranking and the expert ranking are related. When we compare each student-pair ranking with the expert ranking, we find that two teams' rankings generate a Spearman's Rank Statistic of 0.7143 , with a p-value of 0.0331 , which is statistically significant. Considering the rankings of these two teams, $(7,5,2,4,3,6,1)$ and $(7,4,3,5,2,6$, $1)$, with the expert ranking $(6,5,1,7,3,4,2)$ we see that the association is positive. In other 
words, we have only 2 out of 22 teams that begin the course with diagram rankings close to the expert rankings.

\section{Comparing students to one another}

The goal of the third analysis is to compare the students' rankings to one another to see where associations may occur. Since there are 22 student pairs, there are 231 comparisons to be made, using the Spearman Rank Correlation Coefficient. Of these, 38 comparisons were statistically significant. We followed the basic steps in statistical cluster analysis to see if there were distinct groups of students who had similar rankings within the group but different rankings across the groups ${ }^{6}$. In order to better understand how these rankings were related to one another, we started with a "cluster diagram" by representing each ranking with a node and connecting each pair of rankings that were significant (see Figure 2 below). A bold connecting line indicates a p-value less than 0.01 while a regular connecting line indicates a p-value less than 0.05 but greater than 0.01 . We developed the clusters by starting with three nodes that were completely connected, for example, T1, T3, and T11 in Cluster A. Additional nodes were added if they were connected to at least two nodes already in the cluster until all clusters stabilized. The clusters and nonclustered nodes were then organized with respect to their agreement with the expert ranking. The expert ranking is located at the bottom of the diagram and the distance from any node to the expert diagram roughly reflects the level of agreement with the expert ranking.

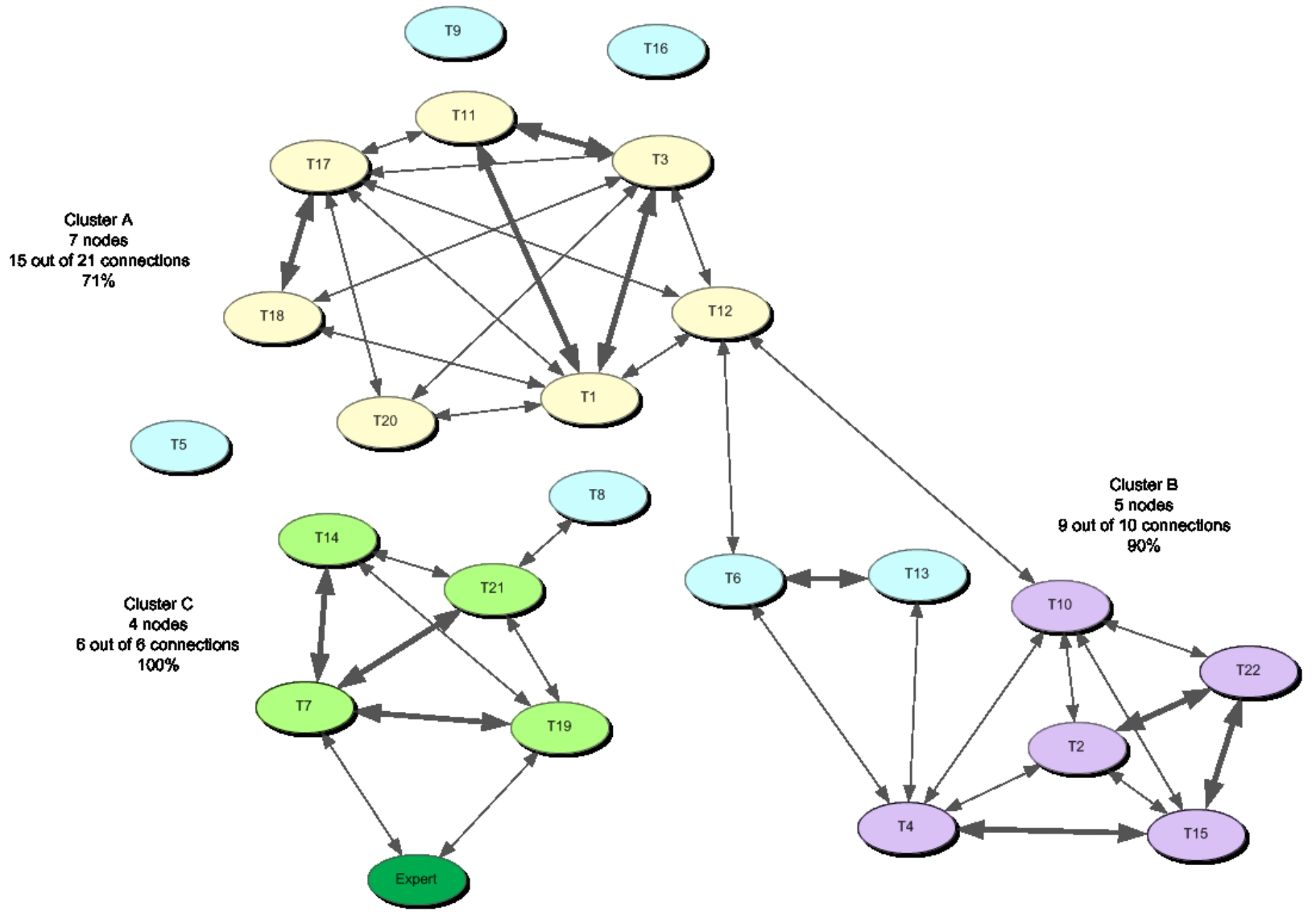

Figure 2: Cluster Diagram Showing Ranking Agreement 
As the diagram illustrates, there are three distinct clusters and six student pairs who are not part of a cluster. A "degree of connectedness" was calculated by comparing the actual number of connections in the cluster to the maximum number of connections. For example, Cluster B has 5 nodes, therefore there are 10 possible connections. Nine of the potential connections are statistically significant; therefore the degree of connectedness for Cluster B is $90 \%$.

Starting at the top of the diagram, we see two student pairs who are not connected to any other pair and are the furthest away from the expert ranking. One other pair, T5, is not connected to any other pair, but this team had more agreement with the expert than did the two teams at the top. Three other teams were connected to one or two teams, but not included in a cluster. Although these teams had a moderate agreement with the expert ranking, the agreements were not statistically significant.

Table 6 below reports the cluster composite rankings, which were computed in the same manner as the class composite ranking, compared to the expert ranking.

\begin{tabular}{|l|c|c|c|c|c|c|c|}
\hline & A & B & C & D & E & F & G \\
\hline Expert & 6 & 5 & 1 & 7 & 3 & 4 & 2 \\
\hline Cluster A & 3 & 5 & 4 & 6 & 1 & 2 & 7 \\
\hline Cluster B & 4 & 5 & 5 & 7 & 2 & 3 & 1 \\
\hline Cluster C & 7 & 4 & 3 & 4 & 2 & 6 & 1 \\
\hline
\end{tabular}

Table 6: Cluster Composite Rankings

Cluster A, near the top of the page consists of 7 student pairs and is $71 \%$ connected. When we compare the cluster composite with the expert ranking, we see that six of these seven teams ranked diagrams $\mathrm{E}$ or $\mathrm{F}$ as their top choice, while the expert ranked them as third and fourth respectively. Diagram G was ranked sixth or last by six of the seven teams; while the expert ranked it second. Overall, students in Cluster A have strong agreement with each other, but there is no evidence of agreement with the expert ranking.

Cluster B, on the right side of the diagram, consists of 5 student teams and is $90 \%$ connected. The composite ranking for Cluster $\mathrm{B}$ resulted in a tie for diagrams $\mathrm{B}$ and $\mathrm{C}$, so they both are assigned rank 5 and there is no diagram with rank 6 . Four of these five student pairs ranked diagram $\mathrm{G}$ as their top choice, which is close to the expert's ranking of it as second. Four of the five also ranked diagram $\mathrm{D}$ as sixth or last, which is in agreement with the expert's ranking as last. All pairs in this cluster ranked the expert's top choice (diagram C) as between fourth and seventh, indicating that they do not agree about this particular diagram.

Cluster C, on the left, at the bottom of the diagram, consists of 4 students teams which are $100 \%$ connected. Two of these teams have statistically significant agreement with the expert ranking. Both the expert and this cluster rank diagrams $\mathrm{C}, \mathrm{G}$, and $\mathrm{E}$ as their top three options. In addition, they both rank diagram A in the last two rankings. Diagram D was the most contested, with the expert ranking it as last $\left(7^{\text {th }}\right)$ and the student teams ranking it between 3 and 5 . 
In summary, our quantitative analysis shows that there are three distinct clusters of student teams who agree with one another and have varying levels of agreement with the expert.

\section{Qualitative analysis of discussion and drawings}

The final analysis uses qualitative data to further explore the meanings of the rankings. During the class period when these data were collected, the first author walked around and observed the students, occasionally asking them questions about their work. The course instructor was the second author. As he conducted the class, he also roamed the room and made observations. These observations and the audiotape of the class discussion provide additional sources of data about the students' process of ranking the Sparkle diagrams and their understanding of diagrams.

As we observed the students beginning the ranking process, we saw that many teams first divided the diagrams into pictures $(A \& F)$ and non-pictures $(B, C, D, E, \& G)$ and then compared the two pictures. This is consistent with the expert/novice literature reporting that novices tend to look at superficial elements when categorizing items ${ }^{2-4}$. Nineteen of the twentytwo teams ranked $\mathrm{F}$ as better than $\mathrm{A}$ and the major difference between the two is $\mathrm{F}$ has one more data item than A. This implies that students recognize the importance of abstracting the data from the problem description.

One team was observed to gather diagrams B, C, and D and set them aside as the "worst" diagrams. These are the only three diagrams with labels for the streams (either letters or numbers inside circles). When asked how they made that decision, a team member responded, "Well, we don't understand what these things in the circles are, so we are calling those the worst." The team did not spend much time brainstorming explanations for "these things" they did not understand and they did not ask for clarification from the instructor or teaching assistants as some teams did. This implies that if students do not understand the notation, icons, or symbols on a diagram, they do not value those items and therefore the diagram.

During the discussion after the ranking activity was finished, the students actively debated the quality of Diagram $\mathrm{G}$, which was the only diagram with the data items listed in a table below the system structure. Nine of the 22 teams ranked this diagram as one of their top two choices; while 8 ranked it as one of their bottom two. The students who liked this diagram spoke of it as "clean, not messy" and asserted that it was "easy to understand". The other side agreed with the expert in wanting the data located strategically and argued that "you can't see where the numbers are used". In cognitive terms, having the data in a table separate from the diagram increases the cognitive load when developing the mathematical models and increases the risk of reading the wrong number from the table. One way to interpret this preference for "clean" diagrams (that is, ones that are less detailed and are simpler) is that more detailed diagrams seem more complex and therefore more difficult, perhaps to the point of invoking anxiety about being able to solve the related problem.

Later in the discussion, one student proposed that some of the diagrams were more "userfriendly" while others were more "engineering." When asked to elaborate, she said that diagrams $\mathrm{A}$ and $\mathrm{F}$ were "nice and more like what you would expect in a user manual" but they really were 
not engineering diagrams. This student demonstrates a tacit understanding of expert criterion number five, which is to be free of distractions.

One issue that did not come up in the discussion was a unique aspect of Diagram C. After the students were told that the expert ranked this diagram as the best, many of them were observed to study this diagram closely. However, no one brought up the fact that this was the only diagram to use noun-noun combinations on the streams, rather than adjective-noun combinations, e.g. clothes and dirt rather than "dirty clothes". Although this seems a trivial difference, it can be very important. We observed that students often did not seem to realize that air contains water and they must decide if the amount of water is worth tracking. The adjective-noun combinations of "hot air" and "humid air" did not provoke a discussion of the water in air, which is a critical part of this part of the problem process - drying the clothes. Without attending to this important aspect of the problem, they would not be able to construct correct mathematical models. This implies that students have difficulty abstracting the species from the text description of the problem.

\section{Implications for Teaching and Learning}

The long-term goal of the larger research agenda is to improve the teaching and learning of conservation principles. This exploratory research has several implications for achieving that goal. First, students begin the course with tacit understandings of what engineering diagrams should look like (recall the discussion around user-friendly versus engineering diagrams); yet they cannot explicitly articulate the features of an effective engineering diagram. This suggests that any engineering course in which diagrams are a critical component explicitly establish with the students the community-sanctioned criteria for evaluating diagrams. In addition to improving their problem solving performance, we hypothesize that explicitly establishing these criteria would improve the students' affective response to the course, and therefore, to the instructor.

Second, the clustering of students' rankings implies that there are different types of prior knowledge and conceptions about diagrams that students bring into the course. Without explicit instruction during class, students may never have these conceptions challenged. Since the pairs in this research and student study groups are self-selected, it is not likely that their initial diagramming practices (e.g. separate the data from the diagram) will evolve into more efficient practices without intentional instruction during class. Since the diagram is the basis for forming the mathematical models, not being able to recognize and generate high quality diagrams would impede students' abilities to construct a correct quantitative solution to the original problem.

Third, textbooks rarely discuss the choices a problem-solver makes in generating a diagram, such as noun-noun combinations for species instead of adjective-noun combinations, or the qualities of a good diagram, such as species balance. This study indicates that students do not enter the course with an explicit awareness of these choices and qualities. In addition, the study indicates that when evaluating a diagram, most students do not use the same criteria as an expert.

Our collective experience strongly suggests that using problems such as the "Wash and Walk" problem enables students to explicitly consider and reform their tacit knowledge and conceptions 
about diagrams as cognitive tools. Understanding community-sanctioned diagrammatic practices increases students' abilities to correctly model and solve mass and energy balance problems.

\section{Directions for Further Research}

This exploratory research has several limitations which should be mitigated in future research studies. First, the problem description and diagrams used in the rankings were developed by the course professor, who also served as the project's primary expert. The quality criteria and expert ranking were constructed by this expert in collaboration with the first author, who is an engineer from a different discipline. Future research studies should ensure that the problem development, the diagram development, and the expert ranking are done by different people.

Second, typical of learning research done in situ, we struggled with balancing the tradeoffs between the best data collection process for student learning and the best process for rigorous research. Having students work in pairs to develop their rankings should require the students to articulate their reasoning and think more deeply about the task. The result is then a negotiated set of rankings that belongs to the pair of students, rather than the individual students, which makes it difficult to analyze the correlation between individuals' performances on assessments with the rankings. We considered giving the ranking task as a homework assignment, but that option has several disadvantages. First, we could not observe them as they performed the task and therefore we would lose a valuable data source. Second, we would not know if they collaborated and if so, with whom. Third, given that this was the first week of class, the students may have felt very anxious about the unfamiliar task, especially if it was part of their grade, which would have decreased the validity of the task as a measure of their understanding.

Third, the details of the data collection could be strengthened. For example, we did not require the students to justify their rankings on paper nor to articulate the criteria they were using. A more carefully constructed set of diagrams may tease out more detail of students' understanding of diagram quality. The students were in self-selected pairs, so prior experience working together could have influenced the way they negotiated the rankings. Random assignment into pairs would strengthen the study. In addition, the method should be replicated with different problems and in different courses to establish the effects of problem and course characteristics.

As in most exploratory studies, in the process of finding answers to our original questions we generated additional interesting questions for future research. First of all, does a student's level of agreement with the expert ranking change over the semester or does it take more time? What learning experiences within the formal curriculum support students learning to recognize good diagrams? Secondly, does the level of agreement with the expert ranking predict performance on assessments such as quizzes, exams, and concept inventories? We are currently investigating these questions. Finally, we connect this research to our original questions by asking whether and how learning community-sanctioned diagrammatic practices will improve students' generation of good diagrams. 


\section{Conclusions}

In conclusion, this exploratory study compared students' and an expert's rankings of a set of diagrams for a complex conservation problem. The quantitative data show that students do not agree with the expert's rankings at the beginning of the semester, yet there are clusters of students who agree with one another. The qualitative data indicate that students are using different criteria than the expert to evaluate the diagrams. In light of these results, we suggest implications for teaching and learning, as well as directions for further research.

\section{Acknowledgements}

This material is based upon work supported in part by the National Science Foundation, under grant number DRL 1109309. Any opinion, finding, conclusions, or recommendations expressed in this publication are those of the authors and do not necessarily reflect the views of the supporting institution. 


\section{Appendix I: Wash \& Walk Problem}

You've come up with a brilliant idea for a start-up business that you call the "Wash and Walk Company". Similar to car wash companies such as the Cactus Car Wash which offers customers full-service car washing, your idea is to provide customers with a full-service real-time clothes washing service. Unlike how you wash clothes at home, your company must develop an industrial clothes washing process that is operated continuously (at steady state) by moving clothes through multiple machines, each of which perform one specific function. In order to minimize keep the price you must charge for this amazing service low, you must determine how to run the process in a way that washes and dries clothes in the most economical manner possible.

Your initial design of the process works as follows: dirty clothes are introduced to the first unit of the system where they are washed with water and soap to remove all the dirt from the clothes. Next, the clean clothes are rinsed with water to remove all the excess soap, after which they are spun (centrifuged) to remove most of the water from the clothes. The wet but clean clothes are then transferred to a dryer, which exposes the clothes to hot air (note: the hot air is generated by a pre-heater which takes air from outside the building and heats it up). Two streams exit the dryer - dry clothes and hot humid air.

a. Below, create a visual representation (diagram, schematic, etc) of the Wash and Walk clothes washing process that would help you quantitatively evaluate its design. Work on your own on this sheet of paper until you receive further instructions.

b. The enigmatic multi-billionaire Sir Yugo Next has decided to invest in your company. Using the funds he has provided, you hire an engineer, Ms. Sparkle Clear, to begin evaluating the process. Sparkle focuses her initial analysis on the dryer and pre-heater units. Sparkle's initial design produces 100 pounds per hour of clean dry clothes. 20 percent of the weight of the wet clothes that enter the dryer is water. The air that enters the preheater is at $70^{\circ} \mathrm{F}$ and has a dewpoint of $50^{\circ} \mathrm{F}$. The preheater uses $2 \mathrm{kw}$ of power to operate and the dryer has a volume of $400 \mathrm{ft}^{3}$. Air exits the preheater (and enters the dryer) at $200^{\circ} \mathrm{F}$, and exits the dryer at $180^{\circ} \mathrm{F}$. The entire process operates at $1 \mathrm{~atm}$ of pressure (i.e., $14.7 \mathrm{psia}$ ). Ms. Sparkle is a newbie engineer and isn't quite sure how to begin, so she produces several diagrams of this part of the process and asks your advice on which diagram she should use to continue her analysis. Examine them and rank order them from best to worst.

c. Consider the part of the process that precedes the part that Ms. Sparkle has diagrammed. Estimate the mass of each component (i.e., mass of clothes, dirt, soap, water, air) of each stream in the process that contains clothes. 


\section{Appendix II: Ms. Sparkle’s Diagrams}

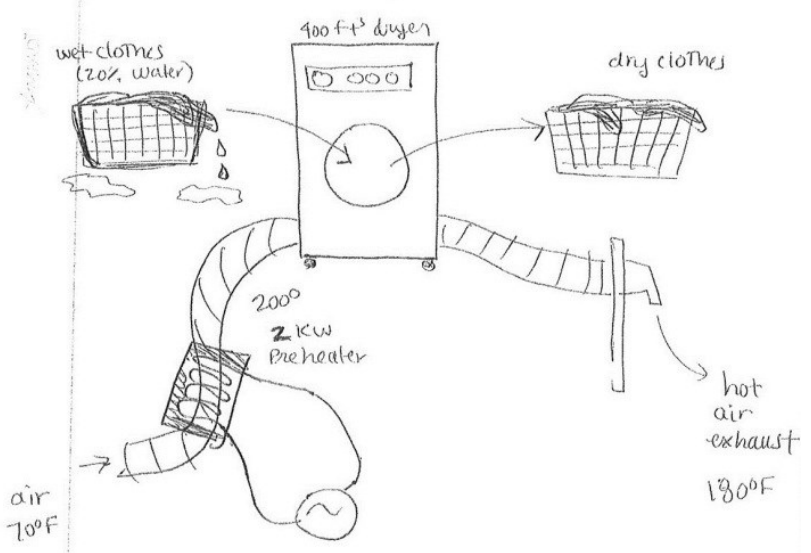

Diagram A

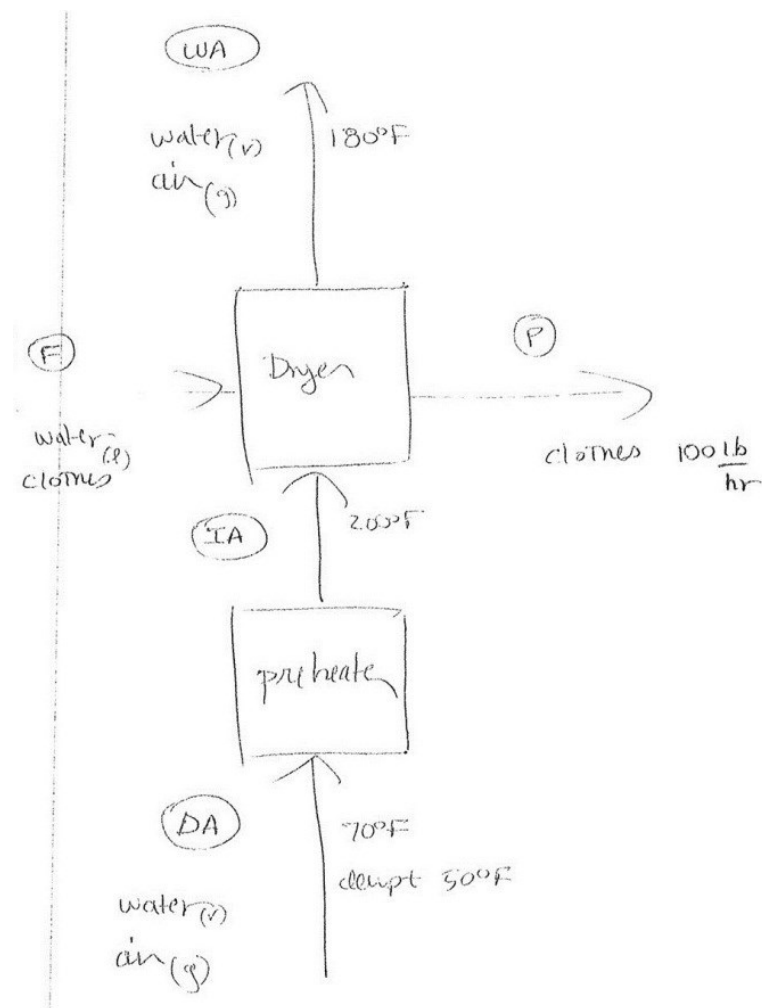

Diagram C

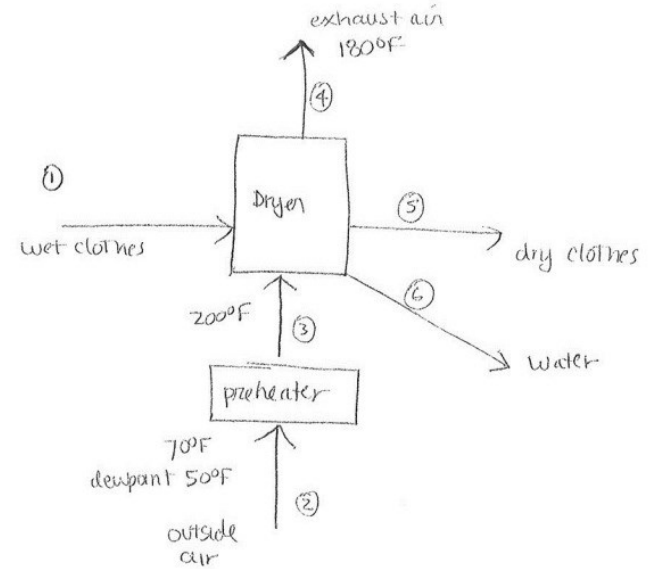

Diagram B

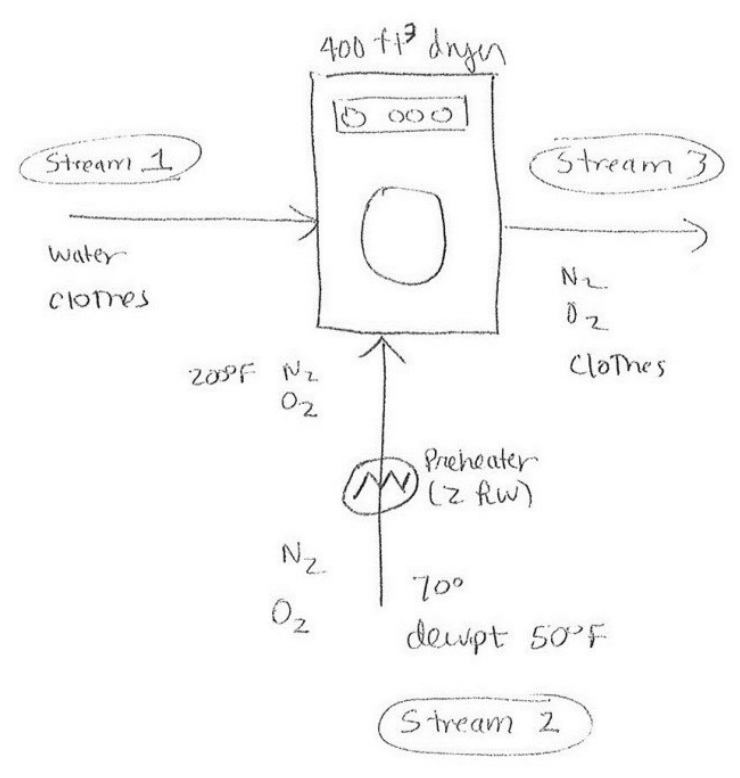

Diagram D

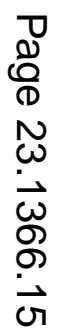




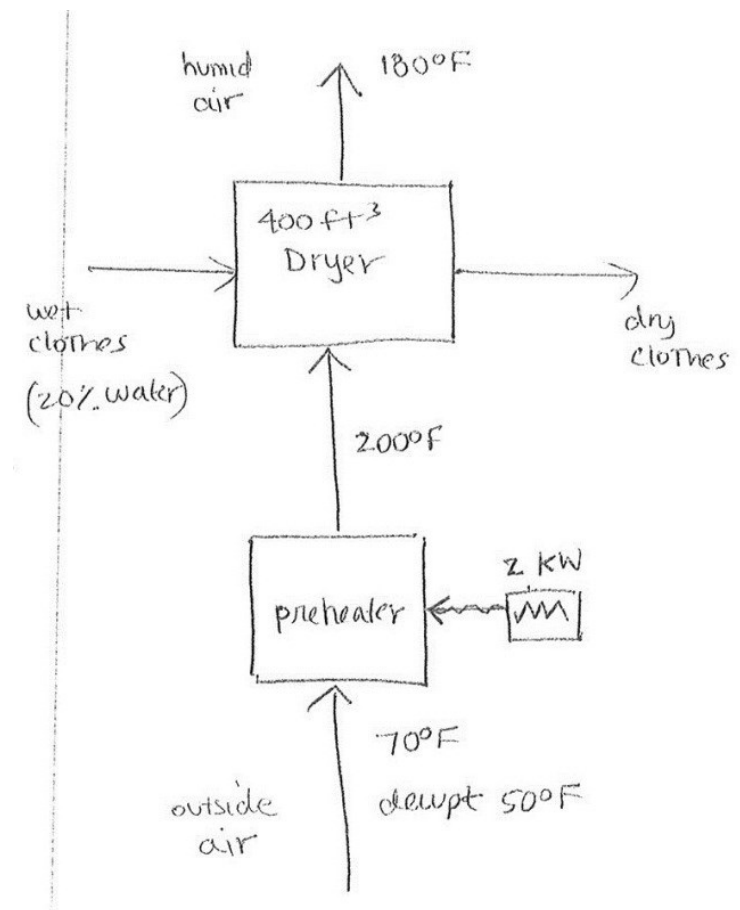

Diagram E

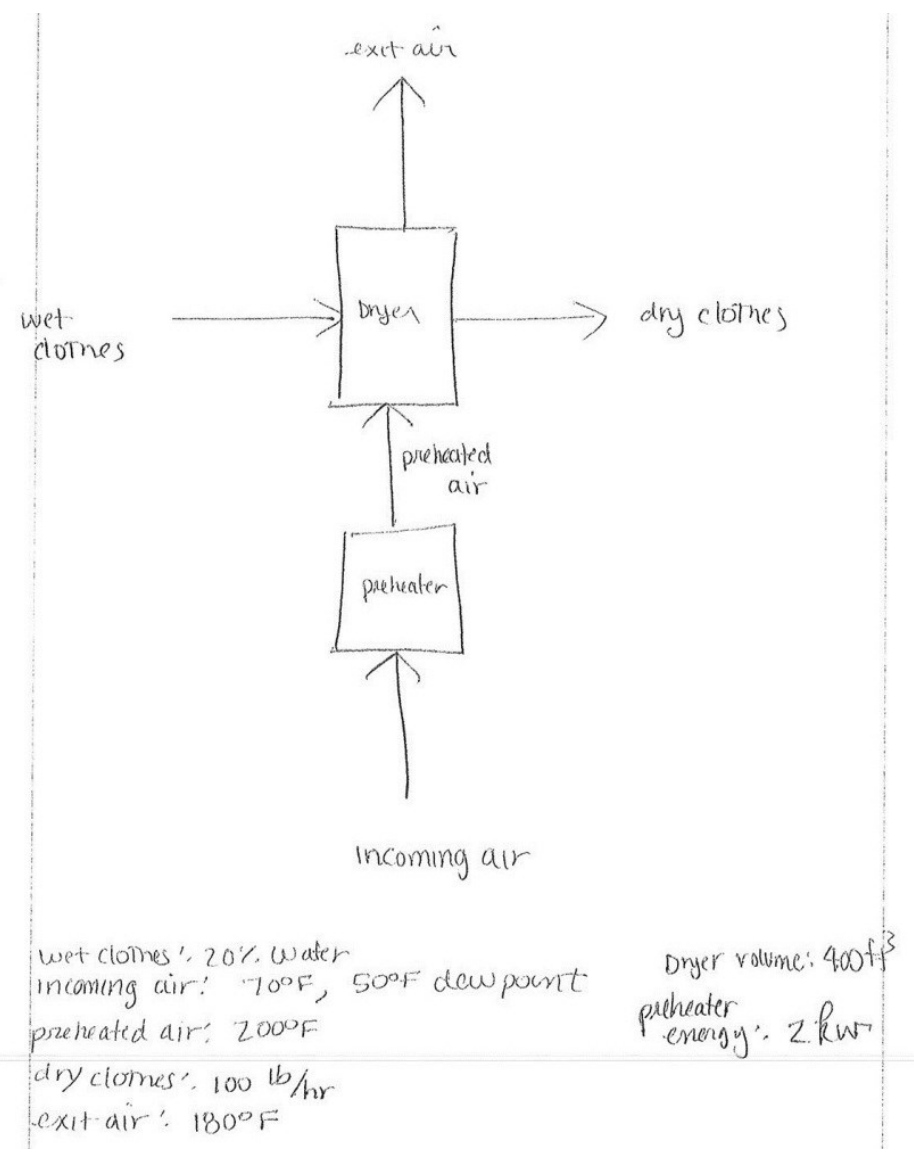

Diagram G

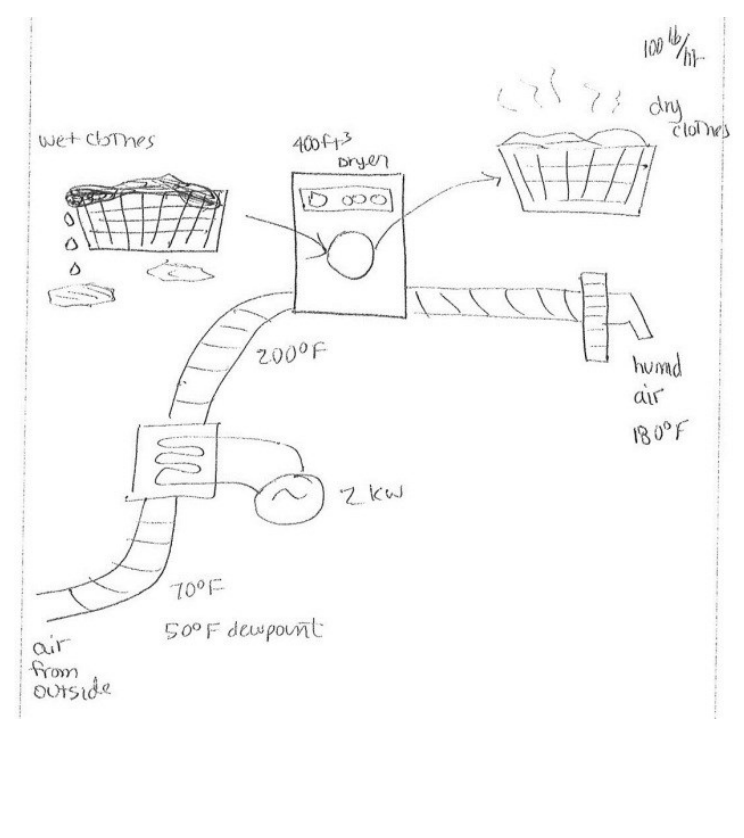

Diagram F 


\section{Bibliography}

[1] W. McCracken and W. Newstetter, "Text to diagram to symbol: Representational transformations in problemsolving," in $31^{\text {st }}$ ASEE/IEEE Frontiers in Education Conf., Reno, NV, 2001, (C) IEEE.

[2] C.J. Atman, et al., "Engineering Design Processes: A Comparison of Students and Expert Practitioners," Journal of Engineering Education, vol. 96, no. 4, pp. 359-379, 2007.

[3] M.T.H. Chi, P. Feltovich, and R. Glaser, "Categorization and representation in physics problems by experts and novices," Cognitive Science, vol. 5, no. 2, pp. 121-152, 1981.

[4] J. Larkin, et al., "Expert and novice performance in solving physics problems," Science, vol. 208, pp. 1335$1342,1980$.

[5] C. Le, Applied Categorical Data Analysis. New York: Wiley, 1998.

[6] J.F. Hair, Jr, et al., Multivariate Data Analysis, Seventh Edition. Upper Saddle River, NJ: Prentice Hall, 2010. 